\title{
STM Study on the Rectifying Performance of Molibdo-Vanadophosphate Polyanions
}

\author{
Sergey A. Kovalevskiy*, Fedor I. Dalidchik, Galina V. Nizova, Evgenii M. Balashov \\ Department of Kinetics and Catalysis, Semenov Institute of Chemical Physics, Russian Academy of Sciences, \\ Moscow, Russia \\ Email: skovalevsk@yandex.ru
}

Received 21 December 2013; revised 21 January 2014; accepted 28 January 2014

Copyright $@ 2014$ by authors and Scientific Research Publishing Inc.

This work is licensed under the Creative Commons Attribution International License (CC BY).

http://creativecommons.org/licenses/by/4.0/

(c) (i) Open Access

\begin{abstract}
Scanning tunneling spectroscopy (STS) was used for investigation of rectification properties of molecules with spatial asymmetry. The molecules of cetyltrimethylammonium derivatives of 10-molibdo-2-vanadophosphate-anions $\left[\mathrm{PMo}_{10} \mathrm{~V}_{2} \mathrm{O}_{40}\right]^{-5}$ (CTMVA) were adsorbed on a surface of pyrolytic graphite (HOPG). Consequently, both good diode properties and J(V)-curves reproducibility of the film were revealed. The validity of the single-level model for the evaluation of the system parameters is discussed.
\end{abstract}

\section{Keywords}

STM, Molecular Diodes, Heteropolyoxometalates, Rectifiers

\section{Introduction}

Single molecular electronics is considered as a promising alternative to conventional electronics towards the ultimate miniaturization of electronic devices. The achievements in this area are reviewed in a number of works [1]-[3]. A considerable attention is devoted to design and research of molecular diodes. Since the fundamental work of Aviram and Ratner [4], plenty of objects were investigated on the basis of organic [5]-[8] and hybrid [9] [10] compounds which demonstrate diode properties. However, the transfer mechanism of electrons in such molecules remains obscure. In the model of Aviram and Ratner [4], charge transfer in molecules containing electron donor (D) and acceptor (A) moieties separated by an insulating bridge (A-b-D molecules) was carried out in the three stages. Under forward bias, electrons can tunnel from one electrode to an acceptor (A), then to donor, and to the second electrode. At the opposite bias, it would require a larger potential to bring the energy levels of the donor and acceptor into favorable alignment with the electrodes. In a number of works [11], it is

*Corresponding author. 
proved that this model is realized. In 2001, the model of the molecular diode based on an asymmetric molecule, where the electroactive part was connected to electrodes through the isolating chains was offered [12]. The asymmetry could be attained by alkyl chains of the different lengths [13] or by various types of links with electrodes, for example at the expense of a chemisorption on the one end of a molecule and physical adsorption on the other end [14] [15]. According to R.S. Williams and all [13], only one electronic level of electroactive part of a molecule participates in electrons transfer. The single-level model recently was used in a number of works for determination of the position of the molecular energy level and coupling to the electrodes. These parameters can be determined from a form of current-voltage dependencies (J(V)-curves) [16] [17]. Recently [18] a new type of molecular diode (organo-inorganic hybrid systems), based on the organoimido derivatives of hexamolybdates, has been designed. The transport of electrons was investigated using density functional theory combined with the non-equilibrium Green's function. High and robust rectification ratio was predicted for these compounds.

The first STM investigation of organo-inorganic hybrid compounds is presented in this work. We study the molecular diode, where the electroactive moiety consists of Keggin's heteropolyanion adsorbed on highly oriented pyrolytic graphite (HOPG) and connected to the other electrode by an alkyl chain. The surface of HOPG is one of the most often used surfaces in the experiments with STM [19]. In this work, the applicability of single-level model is considered. The coupling between CTMVA and a surface of HOPG, and energy gap between HOMO and LUMO orbitals are determined. Similar structures were studied in the work [20] by means of X-ray photoelectron spectroscopy (XPS), scanning tunnel electron microscopy and electrochemical methods. These compounds form ordered structures on the surface of HOPG similar to those observed in [21]-[23] after adsorption of the Keggin-type heteropolyacids. As shown in [20], heteropolyanion is adsorbed on the surface of graphite, and an alkyl chain located above it. The method of film formation is described below.

\section{Results and Discussions}

$1 \mathrm{ml}$ of water solution (50 mg, concentration $25 \mathrm{mM}$ ) containing the excess of $\mathrm{Na}_{2} \mathrm{H}_{3}\left[\mathrm{PMo}_{10} \mathrm{~V}_{2} \mathrm{O}_{40}\right]$ was added to $5 \mathrm{ml}$ of cetyltrimethylammonium bromide $\left[\left(\mathrm{C}_{16} \mathrm{H}_{33}\right) \mathrm{N}\left(\mathrm{Me}_{3}\right)\right] \mathrm{Br}$ in methylene chloride solution $(9 \mathrm{mg}$, concentration $5 \mathrm{mM}$ ). After vigorous stirring for 1 hour the orange-and-yellow precipitate was subsequently transferred to the Eppendorf tube and centrifuged for 2 minutes at 6000 cycles per second. The $20 \mu \mathrm{l}$ of the resulting solution was dropped on the surface of HOPG $(3 \times 5 \mathrm{mM})$. After evaporation the surface was twice washed with deionized water ( $20 \mu \mathrm{l}$ each portion). Then the sample was placed in vacuum for further measurements. The molecules of CTMVA aggregated on a freshly cleaved hydrophobic HOPG surface therefore the surface was previously hydroxylated. For this purpose the sample was introduced in solution of three percent hydrogen peroxide and irradiated for two minutes by $365 \mathrm{~nm}$ UV light. After the pretreatment the surface became hydrophilic with molecules of CTMVA adsorbed on HOPG with $\left[\mathrm{PMo}_{10} \mathrm{~V}_{2} \mathrm{O}_{40}\right]$ anions positioned on the surface and hydrophobic alkyl chains above them. In the course of further study the sample was examined by means of scanning tunnel microscopy that revealed a spot with the adsorbed molecules forming a monolayer structured film. The topographical image coincided with the one displayed in the study [20]. All spectroscopic results were obtained on this film.

Measurements were carried out on STM VT "Omicron" at the room temperature in the ultrahigh-vacuum installation $\left(5 \times 10^{10} \mathrm{mbar}\right)$. Electrochemically etched W tip of STM was cleaned by prolonged argon ions bombardment and current impulses at the voltage of $10 \mathrm{~V}$. During the measurements the tip was brought into the contact with alkyl tails of the adsorbed molecules. Formation of bonds between the tip and alkyl tails of the adsorbed molecule was determined by measuring the dependencies of current on distance between a tip and a surface. The conductance decays exponentially with increasing length. Decay constant $\left(\sim 0.9 \AA^{-1}\right)$ was in accordance with the data obtained for saturated $\sigma$-bonded alkanes. It proves that only the part of alkyl chain between the tip and the surface participates in electron transport. $\mathrm{J}(\mathrm{V})$-dependencies were measured with the currents two order higher than currents prior to the loss of contact of the tip with molecules.

Figure 1 demonstrate $\mathrm{J}(\mathrm{V})$ curves measured on the area $10 \times 10 \mathrm{~nm}^{2}$. The curves are well reproducible. Rectification ratio $\mathrm{RR}=\mathrm{J}(\mathrm{V}+) / \mathrm{J}(\mathrm{V}-)$ reaches maximum value $\mathrm{RR} \cong 15 \div 18$ at the voltage $1.5 \mathrm{~V}$. Good reproducibility most likely is connected with interaction of several alkyl chains with the tip. The part of $J(V)$ curve for negative bias is presented on Figure 2, where the features inherent to all other curves can be seen. The noticeable increase of current occurs at the zero point and at voltage approaching to minus $2 \mathrm{~V}$. Rapid and smooth growth of 


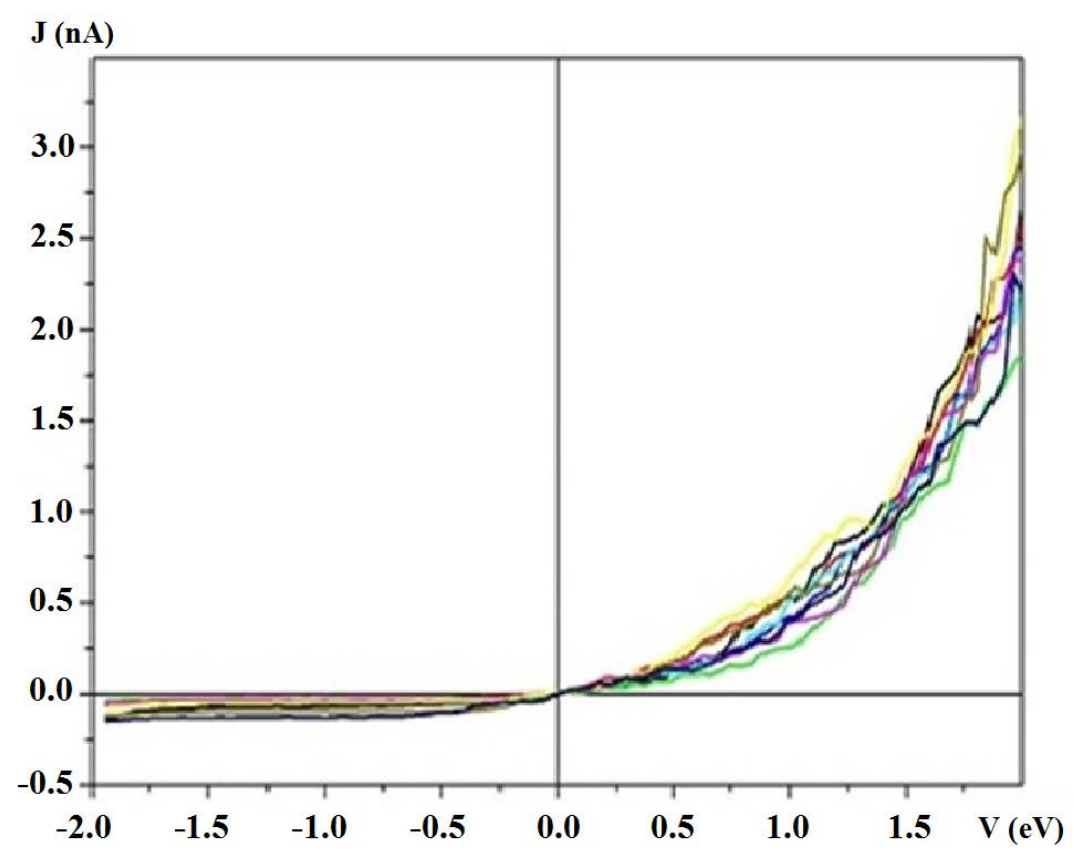

Figure 1. J(V)-curves measured on monolayer film of CTMVA molecules adsorbed on HOPG.

tunnel current with increase of voltage can be observed at a positive bias on a sample.

Molecules under investigation belong to the asymmetric molecules which consist of electroactive moiety and an alkyl chain. Rectification properties of such molecules are actively discussed in a literature. HOMO and LUMO orbitals of electroactive part were used for an explanation of rectification, and it was shown that the location of the electroactive part of molecule within the gap between electrodes is very important [8]. Kornilovich et al. [13] and Baranger et al. [24] considered single-level model where transport of electrons was carried out through molecular orbitals energetically located near Fermi levels of adjacent electrodes and asymmetrically coupled to them. These orbitals could be LUMO [15] or HOMO [24] orbitals. In these works the main attention was paid to definition of parameters that provide the maximum values of rectification ratio. According to the work [13] RR had the maximum values for molecules where the electroactive part was separated from electrodes by the alkyl chains containing two atoms of carbon on one side and 10 atoms on the other side. The authors assumed that the potential drop across the electroactive part of molecular rectifier is insignificant. In the work [24] authors excluded a short chain from model to achieve the maximum current and obtain $R R \cong 10$. They showed that the potential drop across the electroactive moiety is insignificant only for the resonant voltages where molecular level is in alignment with Fermi energy of a lead. Stadler et al. [25] made calculations for various types of molecular diodes, including those proposed by Aviram-Ratner, and concluded that RR can't exceed 20. However, in the latest experiments [26] it was shown that RR reaches 100. The measurements were conducted on self-assembled monolayers of alkanethiolates with head group (SC11Fc). The authors made the conclusion that only one molecular orbital (the HOMO of the Fc) participated in electron transport. Such large rectification ratio is not predicted by theoretical models.

Thus, in these works the main attention was paid to a qualitative explanation of the mechanism of electron transport, but not to attempts of determination of parameters of model by comparison with experimental data. The single-level model allows to restore parameters of molecular orbitals participating in electron transfer (such as, relative position $E_{0}$ of the energy of molecular orbital with respect to the Fermi energy of metal and coupling $\Gamma_{L, R}$ of molecular states to left and right electrodes). This approach developed in works [16] [17] is based on application of Landauer formula for current through metal-molecule-metal junction:

$$
J(V)=\int_{-\infty}^{\infty} T(E, V)\left[f\left(E-\frac{V}{2}\right)-f\left(E+\frac{V}{2}\right)\right] \mathrm{d} E
$$




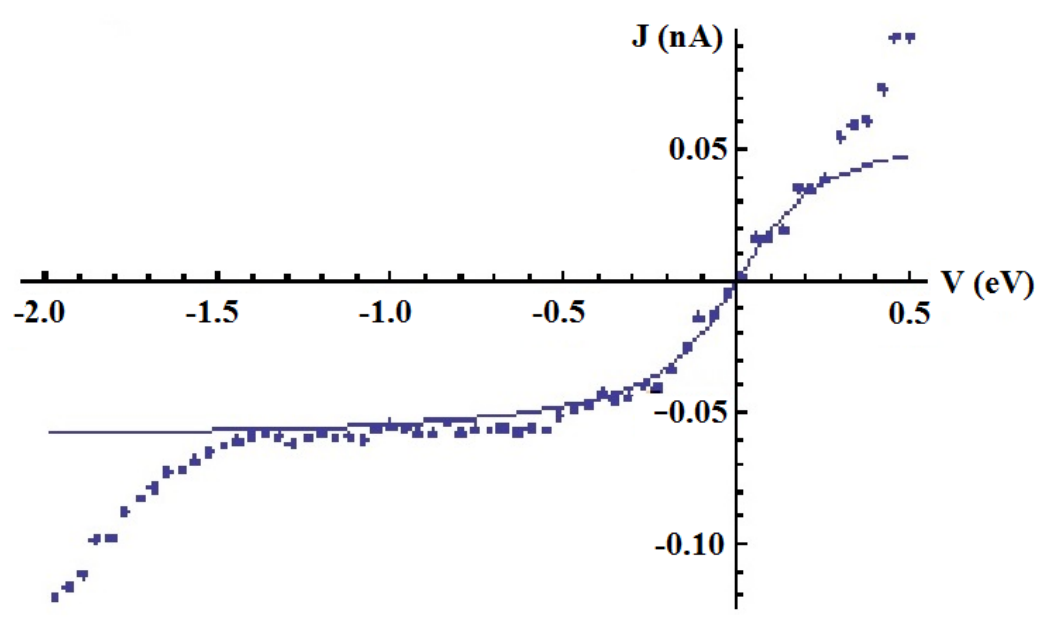

(a)

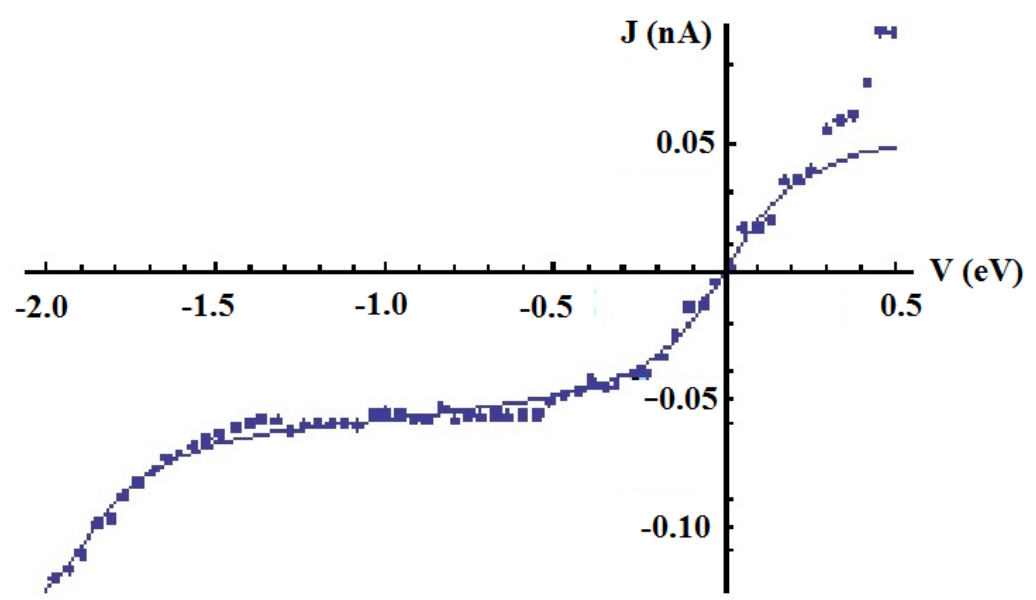

(b)

Figure 2. (a) Experimental (points) and model (line) LUMO J(V)-curves. The parameters used in the fit are: $E_{0}=0 \mathrm{eV} . \Gamma_{R}=0.14 \mathrm{eV}, \Gamma_{L}=0.00000011 \mathrm{eV}$; (b) Experimental and model $\mathrm{J}(\mathrm{V})$-curves. The model curve take into account LUMO and HOMO orbitals. The fitting parameters for HOMO orbitals are: $E_{0}=1.9 \mathrm{eV}$, $\Gamma_{R}=0.18 \mathrm{eV}, \Gamma_{L}=0.00000011 \mathrm{eV}$.

where $T(E, V)$ is the transmission function that depends on the energy $E$ and voltage $V$, $f(E)=1 /[1+\exp ((E-\mu) / k T)]$ is the Fermi function, $\mu$ is the lead chemical potential and $k T$ is the thermal energy. Transmission function in the single-level model is given by the Breit-Wigner formula:

$$
T(E, V)=\frac{4 \Gamma_{L} \Gamma_{R}}{\left(E+E_{0}(V)\right)^{2}+\left(\Gamma_{L}+\Gamma_{R}\right)^{2}}
$$

In the case of asymmetric coupling the position of energy level is a function of the applied voltage. It was assumed [16] that the dependence $E(V)$ on $\Gamma_{L, R}$ can be expressed as:

$$
E_{0}(V)=E_{0}+\frac{e V}{2} \frac{\Gamma_{L}-\Gamma_{R}}{\Gamma_{L}+\Gamma_{R}}
$$

The main assumptions for the validity of this model are coherent tunneling and electron transport through a single molecular resonance in the entire voltage range. The validity of the first assumption seems reasonable for short molecules, the second assumption needs justification. The above-mentioned works [13] [24] [26] justify the applicability of single-level model for an explanation of rectification properties of asymmetric molecules. 
But our attempts to fit experimental J(V) curves to single-level model described by formulas (1)-(3) in the voltage range of $\pm 2 \mathrm{~V}$ were unsuccessful. The reasons of inapplicability of single-level model can be connected with an electronic structure of electroactive moiety of molecules. In the case under consideration the electroactive part consists of Keggin's anion which electronic structure is considered in work [27]. It is known that it consists from a group of free molecular orbitals mostly $\mathrm{d}_{\mathrm{xy}}$ orbitals of metal atoms and group of filled orbitals relating to atoms of oxygen. The size of band gap is approximately $2 \mathrm{eV}$ (for [ $\left.\mathrm{PMo}_{12}\right]$ - moeities LUMOHOMO gap $=2.03 \mathrm{eV}$ ). It is need to take into account that molecules of CTMVA are chemisorbed on hydroxylated hydrophilic graphite surface on one side and connected with the tip through physically adsorbed alkyl chain. Therefore, the electronic structure of Keggin's anion is closely tied with Fermi level of HOPG. Consequently the Fermi level of the tip can be in alignment with a number of closely spaced LUMO levels at the positive bias. On the contrary electrons tunnel from surface to the tip through the nearest LUMO level of heteropolyanion_located slightly above Fermi level of graphite at negative surface bias. For voltages comparable with the band gap, the contribution of HOMO orbital into electron transfer has to be taken into account. Therefore we carried out the fitting using formulas (1)-(3) for negative bias considering only frontier HOMO and LUMO orbitals. The model curve for only LUMO orbital (Figure 2(a)) does not describe the noticeable increase of a current for voltages approaching to minus $2 \mathrm{~V}$. The model curve that takes into account the contribution of HOMO and LUMO orbitals presented on Figure 2(b). Experimental data are well described by this curve. The parameters describing coupling of CTMVA molecules with one electrode have higher values in comparison with parameters of coupling with another electrode.

The histogram of values of the HOMO-LUMO gap obtained from fitting of $54 \mathrm{~J}(\mathrm{~V})$-curves, presented on Figure 3. The measured value of the band gap is in a good agreement with DFT calculations for Keggin's heteropolyacides [26].

\section{Conclusion}

A new type of inorganic-organic hybrid system based on cetyltrimethylammonium derivatives of 10-molibdo-2vanadophosphate-anions $\left[\mathrm{PMo}_{10} \mathrm{~V}_{2} \mathrm{O}_{40}\right]^{-5}$ reveals the good rectification properties. But rectification ratio turned to be lower than RR predicted for organoimido derivatives of hexamolybdates [18]. This may be due to the replacement of a conjugate organic part to an organic part with alkyl chain.

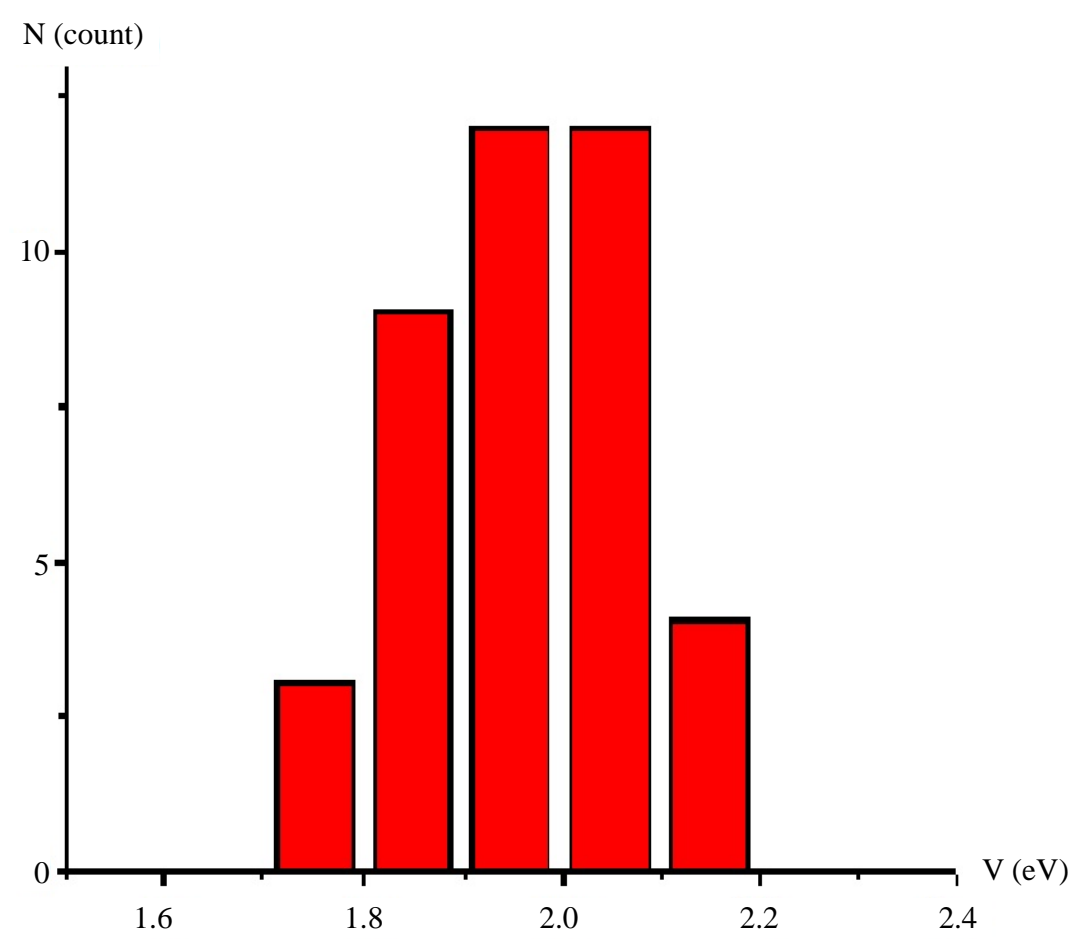

Figure 3. The histogram of HOMO-LUMO bang gap values. 


\section{Acknowledgements}

This work was supported by the Russian Foundation for Basic Research (project №11-03-00494-a).

\section{References}

[1] McCreery, R.L. (2004) Molecular Electronic Junctions. Chemistry of Materials, 16, 4477-4496. http://dx.doi.org/10.1021/cm049517q

[2] Chen, F., Hihath, J., Huang, Z., Li, X. and Tao, N.J. (2007) Measurement of Single-Molecule Conductance. Annual Reviews of Physical Chemistry, 58, 535-564. http://dx.doi.org/10.1146/annurev.physchem.58.032806.104523

[3] Song, H., Reed, M. and Lee, T. (2011) Single Molecule Electronic Devices. Advanced Materials, 23, 1583-1608. http://dx.doi.org/10.1002/adma.201004291

[4] Aviram, A. and Ratner, M.A. (1974) Molecular Rectifiers. Chemical Physics Letters, 29, 277-283. http://dx.doi.org/10.1016/0009-2614(74)85031-1

[5] Chen, A.B. and Metzger, R.M. (1999) Rectification between 370 and 105 K in Hexadecylquinolinium Tricyanoquinodimethanide. The Journal of Physical Chemistry B, 103, 4447-4451. http://dx.doi.org/10.1021/jp990006e

[6] Solomon, A., Cahen, D., Lindsay, S., Tomfohr, J., Engelkes, V.B. and Frisbie, C.D. (2003) Comparison of Electronic Transport Measurements on Organic Molecules. Advanced Materials, 15, 1881-1890.

http://dx.doi.org/10.1002/adma.200306091

[7] Ulgut, B. and Abruna, H.D. (2008) Electron Transfer through Molecules and Assemblies at Electrode Surfaces. Chemical Reviews, 108, 2721-2736. http://dx.doi.org/10.1021/cr068060w

[8] Metzger, R.M., Xu, T. and Peterson, I.R. (2001) Rectification by a Monolayer of Hexadecylquinolinium Tricyanoquino-Dimethanide between Gold Electrodes. Angewandte Chemie International Edition in English, 40, 1749-1752. http://dx.doi.org/10.1002/1521-3773(20010504)40:9<1749::AID-ANIE17490>3.0.CO;2-O

[9] Aswal, D.K., Koiry, S.P., Jousselme, B., Gupta, S.K., Palasin, S. and Yakhmi, J.V. (2009) Hybrid Molecule-on-Silicon Nanoelectronics: Electrochemical Processes for Grafting and Printing of Monolayers. Physika E, 41, 325-344. http://dx.doi.org/10.1016/j.physe.2008.11.001

[10] Joachim, C., Gimzewski, J.K. and Aviram, A. (2000) Electronics Using Hybrid-Molecular and Mono-Molecular Devices. Nature, 408, 541-548. http://dx.doi.org/10.1038/35046000

[11] Wang, B., Zhou, Y., Ding, X., Wang, K., Wang, X., Yang, J. and Hou, J.G. (2006) Conduction Mechanism of Aviram-Ratner Rectifiers with Single Pyridine- $\sigma-C_{60}$ Oligomers. The Journal of Physical Chemistry B, 110, 24505-24512. http://dx.doi.org/10.1021/jp065069t

[12] Krzeminski, C., Delerue, C., Allan, G., Vuillaume, D. and Metzger, R.M. (2001) Theory of Electrical Rectification in a Molecular Monolayer. Physical Review B, 64, 085405. http://dx.doi.org/10.1103/PhysRevB.64.085405

[13] Kornilovitch, P.E., Bratkovksy, A.M. and Williams, R.S. (2002) Current Rectification by Molecules with Asymmetric Tunneling Barriers. Physical Review B, 66, 165436. http://dx.doi.org/10.1103/PhysRevB.66.165436

[14] Taylor, J., Brandbyge, M. and Stokbro, K. (2002) Theory of Rectification in Tour Wires: The Role of Electrode Coupling. Physical Review Letters, 89, 138301. http://dx.doi.org/10.1103/PhysRevLett.89.138301

[15] Zahid, F., Ghosh, A.W., Paulsson, M., Polizzi, E. and Datta, S. (2004) Charging-Induced Asymmetry in Molecular Conductors. Physical Review B, 70, 245317. http://dx.doi.org/10.1103/PhysRevB.70.245317

[16] Zotti, L.A., Kirchner, T., Cuevas, J.-K., Pauly, F., Huhn, T., Scheer, E. and Erbe, A. (2010) Revealing the Role of Anchoring Groups in the Electrical Conduction through Single-Molecule Junctions. Small, 6, 1529-1535. http://dx.doi.org/10.1002/smll.200902227

[17] Kirchner, T., Briechle, B., Scheer, E., Wolf, J., Huhn, T. and Erbe, A. (2012) Statistical Investigation of Current-Voltage Characterization in Single Molecule-Metal Junctions. Acta Physica Polonica A, 121, 410-415.

[18] Wen, S., Yang, G., Yan, L., Li, H. and Su, Z. (2013) Theoretical Study on the Rectifying Performance of Organoimido Derivatives of Hexamolybdates. ChemPhysChem, 14, 610-617. http://dx.doi.org/10.1002/cphc.201200770

[19] Ruben, M., Lehn, J.-M. and Muller, P. (2006) Addressing Metal Centres in Supramolecular Assemblies. Chemical Society Reviews, 35, 1056-1067. http://dx.doi.org/10.1039/b517267p

[20] Tang, Z., Liu, S., Wang, E., Dong, S. and Wang, E. (2000) Adsorption and Electrochemical Oxidation on Carbon of Micelle-Forming Cationic Surfactants Derived from Pyrrole. Langmuir, 16, 5806-5813. http://dx.doi.org/10.1021/la991348e

[21] Kinne, M. and Barteau, M. (2000) STM and TS Investigations of Silver Polyoxometalate Monolayers: Model Compounds and Potential Multifunctional Oxidation Catalysts. Surface Science, 447, 105-111.

http://dx.doi.org/10.1016/S0039-6028(99)01148-6 
[22] Song, I.K., Kaba, M.S. and Barteau, M. (1996) STM Investigation of Pyridine Interaction with Heteropoly Acid Monolayers. The Journal of Physical Chemistry B, 100, 17528-17534. http://dx.doi.org/10.1021/jp960832c

[23] Kaba, M.S., Song, I.K. and Barteau, M. (2002) Site Identification of Mixed Arrays of Keggin-Type Heteropolyacids by Scanning Tunneling Microscopy and Tunneling Spectroscopy. The Journal of Physical Chemistry B, 106, 2337-2342. http://dx.doi.org/10.1021/jp014401s

[24] Liu, R., Ke, S.-H., Yang, W. and Baranger, H.U. (2006) Organometallic Molecular Rectification. Journal of Chemical Physics, 124, 024718. http://dx.doi.org/10.1063/1.2141955

[25] Stadler, R., Geskin, V. and Cornil, J. (2008) A Theoretical View of Unimolecular Rectification. Journal of Physics: Condensed Matter, 20, 374105. http://dx.doi.org/10.1088/0953-8984/20/37/374105

[26] Nijhuis, C.A., Reus, W.F. and Whitesides, G.M. (2010) Mechanism of Rectification in Tunneling Junctions Based on Molecules with Asymmetric Potential Drops. Journal of American Chemical Society, 132, 18386-18401. http://dx.doi.org/10.1021/ja108311j

[27] Maestre, J.M., Lopez, X., Bo, C., Poblet, J.-M. and Pastor, N.C. (2001) Electronic and Magnetic Properties of $\alpha$-Keggin Anions: A DFT Study of $\left[\mathrm{XM}_{12} \mathrm{O}_{40}\right]^{n-},\left(\mathrm{M}=\mathrm{W}, \mathrm{Mo} ; \mathrm{X}=\mathrm{Al}^{\mathrm{III}}, \mathrm{Si}^{\mathrm{IV}}, \mathrm{P}^{\mathrm{V}}, \mathrm{Fe}^{\mathrm{III}}, \mathrm{Co}^{\mathrm{II}}, \mathrm{Co}^{\mathrm{III}}\right)$ and $\left[\mathrm{SiM}_{11} \mathrm{VO}_{40}\right]^{m-}$ $(\mathrm{M}=\mathrm{Mo}$ and W). Journal of American Chemical Society, 123, 3749-3758. http://dx.doi.org/10.1021/ja003563j 